\title{
O Desafio de Projetar Recursos Educacionais com uso de Realidade Virtual e Aumentada
}

\author{
Alexandre Cardoso ${ }^{1}$, Cláudio Kirner ${ }^{2}$, Ismar Frango ${ }^{3}$, Romero Tori ${ }^{4}$ \\ ${ }^{1}$ FEELT - Faculdade de Energia Elétrica - Universidade Federal de Uberlândia (UFU) \\ Av. Joao Naves de Avila, 2121 - 38400-902 - Uberlândia - MG - Brasil \\ ${ }^{2}$ Instituto de Matemática e Computação - IMC \\ Av. BPS, 1303, Bairro Pinheirinho \\ Itajubá - MG - Brasil \\ ${ }^{3}$ Universidade Presbiteriana Mackenzie \\ Programa de Pós-Graduação em Engenharia Elétrica e Computação \\ Rua da Consolação, 930 - 01302-907 - São Paulo - SP \\ ${ }^{4}$ PCS - Departamento de Engenharia da Computação e Sistemas Digitais \\ Av. Prof. Luciano Gualberto, trav. 3 n $^{\circ} .158$ - Universidade de São Paulo \\ São Paulo - SP - Brasil \\ alexandre@ufu.br, ckirner@gmail.com, ismar.silveira@mackenzie.br, \\ rometori@gmail.com
}

\begin{abstract}
This paper discusses the challenges related to the insertion of Virtual and Augmented Reality in the teaching and learning processes, considering their multidisciplinary aspect, the adherence to Web 3.0 and a scenario of possible gains and results with such an insertion. There will be pointed out the features that made VR and AR solutions with a potential big impact, despite not properly explored. as well as the challenges that are presented to Computers and Education field, and possible approaches to solve them.
\end{abstract}

Resumo. Este artigo discute os desafios relacionados à inserção de Realidade Virtual Aumentada nos processos de ensino e aprendizagem, considerando seu caráter multidisciplinar, a aderência à Web 3.0, a adequação da mídia ao conteúdo e o cenário de possíveis ganhos e resultados com tal inserção. Destacam-se as características que tornaram RV e RA soluções com potencial grande impacto, ainda que não devidamente explorado, na educação, bem como os desafios que se apresentam à área de Informática na Educação e possíveis abordagens para solucioná-los.

\section{Realidade Virtual e Aumentada na Educação}

Realidade Virtual (RV) e Realidade Aumentada (RA) se distinguem de mídias tradicionais pela imersão e interatividade que propiciam, com uso de soluções apoiadas por 3D. Ao longo dos últimos anos, tais tecnologias têm merecido atenção pela rápida ascensão mercadológica, na cadeia de Inovação. No Hype Cycle for Emerging Technologies (Gartner's Group, 2016), pode-se verificar que a Realidade Virtual (RV) e Realidade Aumentada (RA) são apontadas como uma das 10 apostas para tecnologias emergentes nos próximos anos: a RV caracteriza-se por possibilitar transportar o 
usuário para uma realidade diferente daquela na qual se encontre, por meio do conceito de imersão, permitindo-o navegar por cenários tridimensionais (ambientes virtuais) disponibilizados em periféricos que suportam a interação em tempo real. A RA se diferencia da RV por manter o usuário em sua própria realidade, porém enriquecendo-a com elementos virtuais 3D interativos. Além da visualização em si, a imersão do usuário em ambientes RV, ou a ampliação da realidade no caso de sistemas RA, pode ser aprimorada com estimulação de outros sentidos, como a audição e o tato. Como diferenciais de utilização da RV e RA em relação a mídias tradicionais, como textos e vídeos, destacam-se:

- motivação de estudantes e usuários, baseada na experiência de $1^{\text {a }}$ pessoa, com retenção da informação vivenciada de forma interativa e pessoal;

- grande poderio de ilustrar características e processos, em relação a outras mídias, com dispensa de grandes arquivos;

- possibilidade de visualizações de detalhes de objetos, desde estruturas microscópicas a universos;

- realização de experimentos virtuais, dispensando necessidade de laboratórios físicos, que pode ser de forma atemporal, fora do âmbito de uma aula clássica;

- aprendizagem ativa, propiciada pela interatividade;

- estímulo à criatividade, catalisando a experimentação;

- oferecimento de igual oportunidade de comunicação para estudantes de culturas diferentes, a partir de representações;

- desenvolvimento de habilidades computacionais e de domínio de periféricos.

Entretanto, há uma série de barreiras a serem contornadas de forma a prover condições de geração de material instrucional, apoiado por RV e RA:

- Geração de Objetos Virtuais: necessidade de modelagem 3D, que requer detida avaliação de demandas, perfis dos objetos virtuais, capacidade de renderização de máquinas destino e aderência dos modelos aos usuários.

- Concepção da Interface Comportamental: Para atingir bom grau de imersão, fazse necessária a concepção da interface, com definição da função de transferência que permita processos interativos de forma natural, dispensando aprendizado de equipamentos e/ou adaptações incômodas. É comum se encontrar soluções nas quais a escolha do periférico tenha se antecipado a definições da solução;

- Aspectos de Imersão: O processo de utilização dos ambientes virtuais e aderência ao ensino/aprendizagem demanda a perda da descrença por parte do usuário na experimentação/navegação/interação. Viabiliza-se tal característica com a imersão do mesmo no ambiente, por meio de aspectos de comportamento desejado, comportamento percebido e atuação motora. O conjunto de software e hardware deve ser adequado à condição de presença do usuário, com processos e reações intuitivos.

\section{Ferramentas de Autoria e Popularização de Geração de Conteúdo}

RV e RA sempre apresentaram desafios para a sua popularização, decorrentes da dependência de dispositivos especiais e de aplicações desenvolvidas por empresas ou especialistas. A forte motivação dessas áreas nas pessoas, advinda do uso de interfaces avançadas e mais intuitivas no espaço tridimensional (3D), não foi suficiente para superar as limitações e torná-las popular.

No entanto, com a evolução tecnológica, vários elementos encontrados em dispositivos especiais de RV e RA passaram a fazer parte de smartphones. Além disso, surgiram capacetes de plástico e de papelão, sem eletrônica e de baixo custo ou mesmo 
custo zero, que podiam ser adquiridos ou construídos pelo próprio usuário para incorporar smartphones. Assim, um dos desafios da popularização de RV e RA, relacionados com a disponibilidade e com o custo dos dispositivos especiais, estava resolvido.

Restava ainda o desafio da disponibilização de aplicações de RV e RA adequadas às necessidades dos usuários. O que se tem majoritariamente, hoje em dia, ainda são aplicações desenvolvidas por empresas e especialistas, que satisfazem mais a curiosidade das pessoas, pelo seu aspecto lúdico, do que suas reais necessidades.

$\mathrm{Na}$ área educacional, as demandas por aplicações personalizáveis são muito fortes, em função das características regionais e culturais dos professores e estudantes. A maneira mais apropriada de permitir a personalização das aplicações é ter-se ferramentas de autoria de aplicações adequadas para não especialistas e aplicações customizáveis com facilidade de troca de conteúdo. Desta maneira, professores e estudantes poderão desenvolver e apropriar-se de suas próprias aplicações, disponibilizando-as em repositórios abertos para uso por qualquer interessado, popularizando assim, o desenvolvimento, adaptação e uso de aplicações de RV e RA.

Nesse sentido, à guisa de exemplo, relatam-se aqui alguns aspectos do desenvolvimento e evolução de uma ferramenta de autoria de aplicações de RA e RV, denominada FLARAS (Souza, Moreira, Kirner, 2013), que permite, a usuários não especialistas em informática, definir pontos no espaço 3D e colocar informações e objetos 3D sobre eles. Além disso, esses elementos podem ser configurados, sonorizados com sons e narrações, animados e receberem funcionalidades interativas. No contexto do desenvolvimento do FLARAS, surgiu outro entrave para não especialistas: Como produzir as informações e objetos 3D que irão povoar o ambiente 3D das aplicações de RV e RA? Primeiramente, deve-se considerar que, embora lúdico, muitas vezes os objetos $3 \mathrm{D}$ são desnecessários no ambiente $3 \mathrm{D}$, podendo ser substituídos por textos e imagens, facilitando assim o desenvolvimento da aplicação e sua utilização por ficar mais "leve". Uma característica importante das aplicações com FLARAS é que o ambiente virtual interativo fica sobreposto com o ambiente real capturado pela câmera nas aplicações de RA. Ao obstruir-se a câmera, o ambiente virtual permanece na tela, constituindo uma aplicação de RV.

Uma versão da ferramenta FLARAS para dispositivos móveis também foi desenvolvida, constituindo a ferramenta de autoria de aplicações de RA e RV denominada MAREAS. Essa ferramenta, ainda em fase de testes e de produção de tutoriais, deve ser disponibilizada oportunamente para uso gratuito no site de RV mencionado em Kirner (2017). O desenvolvimento de de aplicações de RA e RV ocorre através de toques na tela do smartphone, usando recursos carregados por download ou transferência para o dispositivo.

Finalmente, um grande desafio para a área é tornar as aplicações de RV e RA inclusivas para pessoas com deficiência, envolvendo principalmente deficientes visuais e auditivos. Tanto o FLARAS quanto o MAREAS possuem recursos para apoiar essas pessoas. Para os deficientes visuais, FLARAS se serve de ambientes de baixo relevo, cujos pontos em protuberância são ativáveis pelo usuário e percebidos pela aplicação através de visão computacional, emitindo retorno sonoro. Para os deficientes auditivos, FLARAS usa retorno de texto e imagens com maior intensidade. Esses ambientes com retornos sonoros e textuais, além de servir para a integração dos deficientes a grupos de usuários sem deficiência, também permite estimular o uso de aplicações por crianças e idosos, compensando a falta ou a perda de destreza na interação com a aplicação. Já a 
MAREAS usa retorno sonoro para deficientes visuais e retorno de texto e de imagens para deficientes auditivos, baseando-se na ativação por posicionamento na tela de toque do celular. Essas aplicações podem ser customizadas pelos usuários, trocando conteúdo envolvendo principalmente imagens, textos, sons e vídeos, podendo inclusive realizar conversão para outras Línguas, dando assim maior alcance às aplicações.

Portanto, apesar da popularização da RV e da RA ter sido um grande desafio, percebe-se que ferramentas de autoria e abordagens apropriadas podem contribuir para o desenvolvimento e adaptação de aplicações, de forma a atingir um grande número de usuários de forma personalizada, incluindo aqueles que apresentam alguma dificuldade de uso, apoiando principalmente a modernização da Educação.

\section{Conteúdo Significativo e Interativo}

Sempre que surge uma nova mídia é comum que professores e criadores de material instrucional corram para desenvolver ou adaptar conteúdos para essa nova tecnologia. Em geral ocorre uma certa euforia no início, com corpos docente e discente motivados com a novidade. Artigos e palestras em eventos enaltecem a chegada daquela que parece ser a tecnologia educacional definitiva, com resultados promissores de experimentos, nos quais invariavelmente aparecem avaliações positivas e entusiasmadas dos participantes. Novas startups surgem, como por exemplo a Oculearn (Swift and Allatt, 2017), especializada no desenvolvimento de conteúdos educacionais de RV. Toda essa euforia, contudo, pode se transformar em frustração e até mesmo aversão à mídia que parecia ser a solução para todos os problemas.

Várias são as questões e desafios envolvidos no uso de mídia em educação (Tori, 2010). A primeira questão é que o ser humano gosta de novidade. Isso explica porque os primeiros experimentos de uso de uma nova mídia invariavelmente conseguem envolver alunos e professores e são bem avaliados. Esses bons resultados incentivam a adoção crescente dessa novidade, muitas vezes, com grande foco na mídia e pouco no conteúdo. Ao deixarem de ser novidade os conteúdos que não forem excelentes tornamse tediosos e o desgaste provocado pelos conteúdos menos interessantes acaba por prejudicar a aceitação até mesmo daqueles melhores. Outro erro comum são comparações entre "aulas convencionais" e "aulas produzidas com a nova mídia". Essas comparações dificilmente conseguem separar mídia de metodologia. Além disso, as condições e os investimentos em preparação são discrepantes, o que compromete qualquer experimento científico, sem falar nos fatores "novidade" e "interesse artifical" que os experimentos com alunos produzem.

É inegável o potencial da RV e da RA como forma de tornar os conteúdos mais imersivos e eficazes a custos menores que transportar fisicamente os alunos para outras realidades ou criar réplicas e/ou simulações físicas de fenômenos. Mas para que os conteúdos virtuais imersivos sejam bem sucedidos é importante que alguns requisitos sejam atendidos:

- Significado: Os conteúdos devem ser significativos para os alunos, ou seja, devem fazer sentido e se relacionar com seus conhecimentos. É importante que o aprendiz entenda a importância daquilo que irá aprender e vislumbre como isso pode impactar a sociedade ou sua futura profissão. $O$ interesse deve ser pelo conteúdo, não pela mídia. Essa irá apenas torná-lo fácil e agradável de ser aprendido, mas não tem capacidade de torná-lo significativo.

- Interatividade: A interatividade é o potencial de interação percebido pelo usuário de determinado sistema (Tori, 2010). Mesmo que o aluno não precise interagir o tempo todo, o fato de saber que pode interagir faz toda diferença. Isso é interatividade, a 
qual reduz a distância entre aluno e conteúdo (Tori, op. cit.). Se o aprendiz não se sentir próximo ao conteúdo de RV ou RA a aprendizagem ficará aquém do potencial que a mídia, o conteúdo e a metodologia oferecem. A interatividade também deve ser significativa. $\mathrm{O}$ interator precisa perceber o impacto de suas ações e sentir que essas causam interferências importantes e significativas ao sistema.

- Qualidade: Além da qualidade técnica do conteúdo desenvolvido para RV e RA (não pode haver atrasos, distorções, erros etc..), o que, por si já é um grande desafio, deve haver qualidade no design, na narrativa e na interface. A qualidade pedagógica nem precisa ser citada porque essa é condição necessária e indispensável. Para a criação de realidades verossímeis é indispensável o cuidado com a narrativa (a experiência do cinema e dos games pode ajudar) e evitar fatores que lembrem o participante de que se encontre em outra realidade. A interface tem um papel importante nesse ponto. Sair da imersão para acionar menus e comandos da forma tradicional quebram o realismo e a imersão. Daí a importância de se criar formas de interação integradas ao ambiente virtual e o uso de interfaces naturais. Um dado importante é que a fidelidade gráfica da representação dos ambientes e personagens não é o fator mais relevante (Jerald, 2016).

\section{Reuso de Recursos Educacionais com suporte a RV e RA}

Por fim, uma discussão sobre os aspectos de reúso de recursos educacionais se faz necessária, dentro do âmbito de aplicações educacionais de RV e RA. Nos anos iniciais da pesquisa em Objetos de Aprendizagem (OA), acreditava-se haver uma relação direta entre a granularidade dos OAs e seu potencial de reuso (Silveira et al., 2007). Esta convicção era possivelmente influenciada pelas experiências de reúso no âmbito da Engenharia de Software - em especial no que tange ao desenvolvimento orientado a componentes, mas a evolução da área veio a mostrar que tal correlação nem sempre é observada, uma vez que no campo educacional, o contexto no qual os OAs se inserem desempenha papel extremamente importante, o que torna o real reuso de OAs fora de seu contexto uma tarefa mais complexa.

Novas abordagens vêm surgindo por meio da aplicação de princípios de abertura ao design de OAs, no âmbito do movimento REA (Recursos Educacionais Abertos) (Wiley e Green, 2012). Ao adotar os princípios 5R - Reúso, Revisão, Remixagem, Redistribuição e Retenção de licença (Wiley, 2014), os REA podem aumentar o potencial de reuso dos recursos educacionais, uma vez que reúnem as características necessárias a diferentes tipos de adaptação e modificação que se requeiram. Entretanto, isto não é facilmente quantificável, o que leva a um desafio específico no contexto dos REA, como aponta Silveira (2016), que é a superação da falta de evidência de reúso dos REA. Beaver (2013) alerta que o reuso de REAs não é facilmente rastreável, uma vez que ele pode se dar de maneira indireta (como inspiração na criação de outros REAs) ou ainda em situações de repurposing.

Ao se especializar esta discussão no que tange aos recursos educacionais com suporte interfaces mídias (ainda) não-convencionais, dois desafios principais, específicos relacionados ao reuso surgem. $\mathrm{O}$ primeiro deles é relacionado à revisão, remix e repurposing de REAs com suporte a RA/RV: o suporte a alterações constantes é característica inerente aos REA. Ao suportar interfaceamento com RV e RA, esta característica converte-se em um desafio tanto técnico quanto ao que diz respeito ao processo de design instrucional. Há níveis crescentes ou decrescentes de complexidade quando se trata de promover mudanças em conteúdos projetados para suportar RV e RA. A Figura 1 exibe um continuum de complexidade em relação aos diferentes graus 
de mudança possíveis em conteúdo educacional desta natureza.

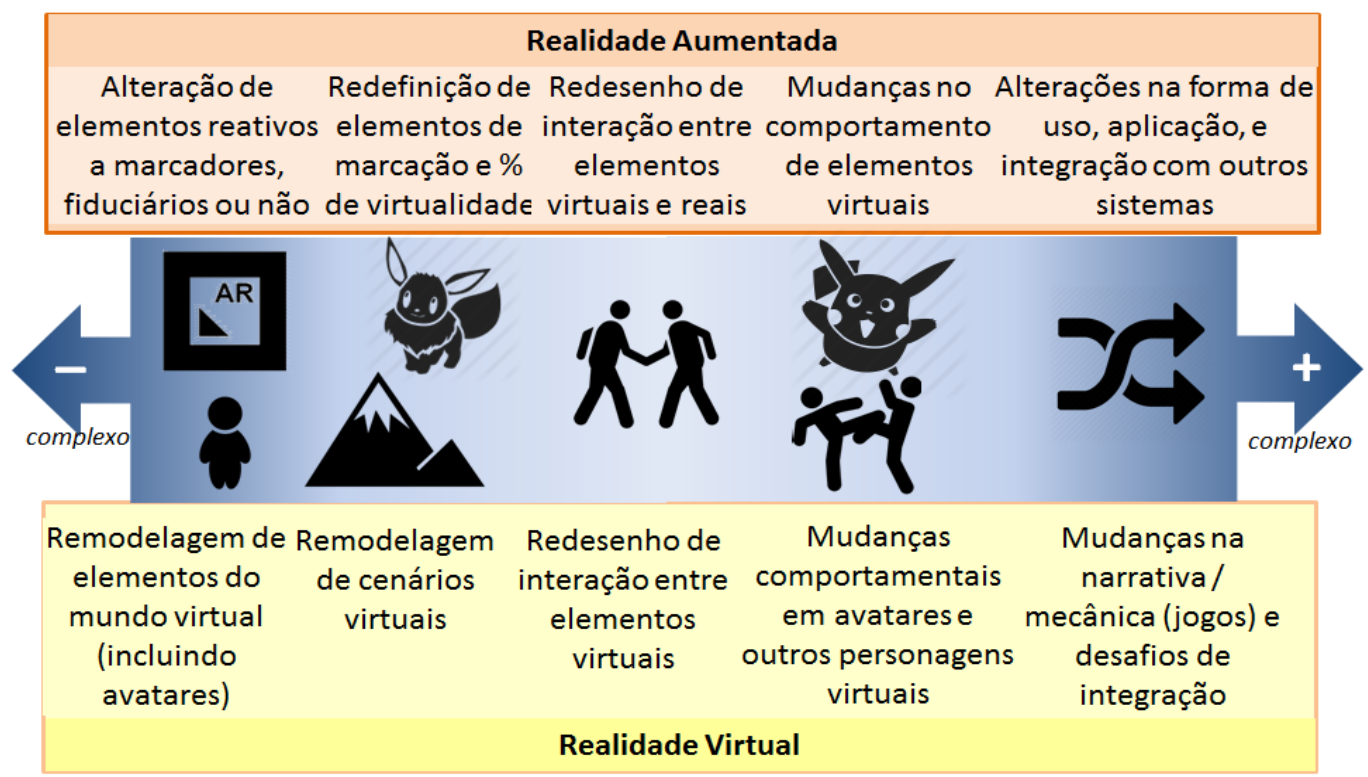

Figura 1. Continuum de complexidade de realização de alterações em REA com suporte a RA e RV. (Fonte: autores)

$\mathrm{O}$ segundo desafio refere-se ao reuso entre dispositivos. Um dos principais problemas no que tange ao reuso de aplicações está na incompatibilidade entre dispositivos. No contexto educacional, o desenvolvimento de recursos digitais com suporte a RA e RV frequentemente está intrinsecamente conectado a um determinado dispositivo, ou ainda a uma família bem-definida destes. Nesse sentido, iniciativas como a OSVR - Open-source Virtual Reality (Boger, Pavlik e Taylor, 2015) lançam luz sobre este problema, propondo uma arquitetura que permita a interoperabilidade de conteúdo, ainda que desenvolvidos para dispositivos específicos.

\section{Proposta de Metas e Ações para Enfrentar o Desafio}

O Quadro 1 sintetiza, nas duas primeiras colunas, um conjunto de metas e ações para enfrentar o desafio e, na última coluna apresenta formas para monitoramento e avaliação do cumprimento das metas e ações para a próxima década.

Quadro 1: Metas, Ações e Monitoramento.

\begin{tabular}{|c|c|c|}
\hline Metas & Ações & $\begin{array}{c}\text { Monitoramento e } \\
\text { Avaliação }\end{array}$ \\
\hline \multirow{2}{*}{$\begin{array}{l}\text { Geração de } \\
\text { recursos } \\
\text { educacionais, } \\
\text { baseados em RV e } \\
\text { RA }\end{array}$} & $\begin{array}{l}\text { Constituição de equipes multidisciplinares } \\
\text { para apoio e acompanhamento de projetos }\end{array}$ & $\begin{array}{llr}\text { Avaliar o } & \text { resultado da } \\
\text { aderência } & \text { do material } \\
\text { produzido. } & & \\
\end{array}$ \\
\hline & $\begin{array}{l}\text { Disseminação de ferramentas de autoria, que } \\
\text { dispensam aprendizado de etapas demoradas } \\
\text { e de programação }\end{array}$ & $\begin{array}{l}\text { Avaliar a utilização das } \\
\text { mesmas e as demandas por } \\
\text { aprimoramento. }\end{array}$ \\
\hline \multirow{3}{*}{$\begin{array}{l}\text { Garantia de } \\
\text { qualidade do } \\
\text { conteúdo de RV e } \\
\text { RA }\end{array}$} & $\begin{array}{l}\text { Estabelecimento de padrões de qualidade } \\
\text { técnica dos ambientes e dispositivos }\end{array}$ & $\begin{array}{l}\text { Avaliação da qualidade } \\
\text { técnica e sua aderência aos } \\
\text { padrões }\end{array}$ \\
\hline & $\begin{array}{l}\text { Estabelecimento de diretrizes para o } \\
\text { desenvolvimento de conteúdos significativos } \\
\text { e adequados }\end{array}$ & $\begin{array}{l}\text { Avaliação da aderência dos } \\
\text { conteúdos às diretrizes }\end{array}$ \\
\hline & Estabelecimento de heurísticas & Aplicação \\
\hline
\end{tabular}




\begin{tabular}{|c|c|c|}
\hline & $\begin{array}{l}\text { avaliação da experiência do aluno (interface, } \\
\text { interatividade, imersão, usabilidade etc.) }\end{array}$ & $\begin{array}{l}\text { para avaliação } \\
\text { experiência do aluno }\end{array}$ \\
\hline \multirow{2}{*}{$\begin{array}{l}\text { Popularização da } \\
\text { RV e RA }\end{array}$} & $\begin{array}{l}\text { Concepção de ferramentas de autoria para } \\
\text { não especialistas }\end{array}$ & $\begin{array}{l}\text { Aferição da facilidade de } \\
\text { uso, aceitação da ferramenta } \\
\text { pelos potenciais usuários e } \\
\text { clientes }\end{array}$ \\
\hline & $\begin{array}{l}\text { Popularização de material gerado, em RV e } \\
\text { RA, por meio de canais de divulgação e } \\
\text { Web }\end{array}$ & $\begin{array}{l}\text { Verificação do aumento da } \\
\text { audiência. }\end{array}$ \\
\hline \multirow{2}{*}{$\begin{array}{l}\text { Reúso e } \\
\text { Personalização das } \\
\text { aplicações e seus } \\
\text { conteúdos }\end{array}$} & $\begin{array}{l}\text { Facilitar compartilhamento de conteúdo; } \\
\text { facilitar aprendizagem da concepção de } \\
\text { novas aplicações }\end{array}$ & \multirow[t]{2}{*}{$\begin{array}{l}\text { Mensuração da facilidade de } \\
\text { compartilhamento e de } \\
\text { criação de novos recursos. }\end{array}$} \\
\hline & $\begin{array}{l}\text { Criação de repositórios abertos de aplicações } \\
\text { e conteúdos educacionais para RV e RA. }\end{array}$ & \\
\hline
\end{tabular}

Vale ressaltar que este quadro pretende-se uma síntese, não exaustiva, das discussões levantadas com maior detalhe no corpo deste artigo.

\section{Considerações Finais}

Apontadas como promessas computacionais eficazes na aproximação e estreitamento do diálogo entre os agentes dos processos de ensino e aprendizagem, Realidade Virtual e Aumentada podem auxiliar, sobremaneira, alguns desafios de tais processos.

Entretanto, como pôde-se relatar, há diversos aspectos fundamentais que serão colocados como desafios para a disseminação de tais tecnologias. Os autores apontam, para a próxima década, a possibilidade de popularização de recursos educacionais por tais agentes, com dispensa de aprendizagem de técnicas e métodos complexos de programação, a geração de conteúdo significativo e o reuso de recursos educacionais já concebidos e em bom uso como elementos de tais processos, tendo os princípios de abertura como norteadores para um efetivo reúso.

Há um conjunto de desafios importantes a serem enfrentados pelas comunidades científica e de prática; o Quadro 1, apresentado na última seção deste artigo, buscou sintetizar os principais direcionamentos para o avanço do estado da arte no que tange à concepção de recursos educacionais com suporte a RV e RA. Os autores esperam, com isso, haver contribuído para as discussões a respeito deste tópico, cuja importância na área de Informática na Educação tende a crescer de maneira ainda mais significativa nos anos vindouros, considerando todos os avanços tecnológicos e a crescente pervasividade de dispositivos e soluções computacionais no campo de RV e RA.

\section{Referências}

Boger, Y. S., Pavlik, R. A., \& Taylor, R. M. (2015). "OSVR: An open-source virtual reality platform for both industry and academia”. IEEE Virtual Reality, p. 383-384.

Gartner Group (2016) "Emerging Technology Hype Cycle". Disponível em: http://blogs.gartner.com/smarterwithgartner/files/2016/08/Emerging-TechnologyHype-Cycle-for-2016_Infographic_revise2.jpg, Acesso em: 17/04/2017.

Jerald, J. (2016) The VR Book: Human-Centered Design for Virtual Reality. Association for Computing Machinery and Morgan \& Claypool Publishers, 2016.

Kirner, C. (2017). Realidade Virtual e Aumentada, 2017, Disponível em: http://realidadevirtual.com.br. Acesso em: 18/04/2017. 
Silveira, I. F., Araújo, C. F., Amaral, L. H., Alcântara de Oliveira, I. C., Schimiguel, J., Ledôn, M. F. P., \& Ferreira, M. A. G. V. (2007). "Granularity and reusability of learning objects". In: Learning objects and instructional design, v. 1, 139-170.

Silveira, I. F. (2016). "OER and MOOC: The need for openness". Issues in Informing Science and Information Technology, 13, 209-223.

Souza, R.C., Moreira, H.D.F. Kirner, C. (2013) FLARAS - Flash Augmented Reality Authoring System. Disponível em: http://ckirner.com/flaras2. Acesso em: 18/04/2017.

Swif, R. and Allatt, D. (2017) Virtual Reality In Education: Our Path to Reality. London: Oculearnig.

Tori, R. (2010) Educação sem Distância. São Paulo: Editora Senac, 2010.

Wiley, D. (2014). The Access Compromise and the 5th R. Iterating toward openness. Disponível em https://opencontent.org/blog/archives/3221. Acesso em: 13/4/2017.

Wiley, D., \& Green, C. (2012). "Why openness in education”. Game changers:

Education and information technologies, p. 81-89. 